\title{
Doppler Echocardiography Inaccurately Estimates Right Ventricular Pressure in Children with Elevated Right Heart Pressure
}

\author{
Georgeann K. Groh, MD*, Philip T. Levy, MD*, Mark R. Holland, PhD ${ }^{\dagger}$, Joshua J. Murphy, \\ MD*, Timothy J. Sekarski, RDCS $^{*}$, Craig L. Myers, RCCS ${ }^{\star}$, Diana P. Hartman, RDCS ${ }^{*}$, \\ Rebecca D. Roiger, MSN, RN, CPNPף, and Gautam K. Singh, MD* \\ "Department of Pediatrics, Washington University School of Medicine, St. Louis, Missouri \\ tDepartment of Physics, Washington University, St. Louis, Missouri \\ ISt. Louis Children's Hospital, St. Louis, Missouri
}

\section{Abstract}

\begin{abstract}
Background-Doppler echocardiography (DE) is widely used as a surrogate for right heart catheterization (RHC), the gold standard, to assess and monitor elevated right heart pressure in children. However, its accuracy has not been prospectively validated in children. The objective of this study was to evaluate the accuracy of DE in predicting simultaneously measured RV pressure by RHC in pediatric patients, and to determine if the degree of RV hypertension affects the accuracy of DE in assessing right heart pressure.
\end{abstract}

\begin{abstract}
Methods-Eighty children (age 0-17.9 years, median 5.5 years) with two-ventricle physiology and a wide range of right heart pressures underwent simultaneous DE and RHC. The pressure gradient between the right ventricle and right atrium was directly measured by RHC and simultaneously estimated by DE using tricuspid valve regurgitation. Patients were then grouped based on RHC measured right ventricular systolic pressure (RVSP): group $1(n=43)$ with RVSP $<1 / 2$ systemic systolic blood pressure (SBP); group $2(n=37)$ with RVSP $\geq 1 / 2$ SBP; group 3 $(n=56)$ with RVSP <2/3 SBP; and group $4(n=24)$ with RVSP $22 / 3$ SBP. Correlation and BlandAltman analyses were performed on all groups. Accuracy was predefined as $95 \%$ limits of agreement within $\pm 10 \mathrm{mmHg}$.
\end{abstract}

Results-Despite a reasonable correlation between DE and RHC in all groups, there was poor agreement between techniques as RVSP/SBP increased. DE was inaccurate in 1/43 (2\%) patients in group 1 versus $9 / 37$ (24\%) in group 2, and was inaccurate in 1/56 (2\%) in group 3 versus 8/24 $(33 \%)$ in group 4 . Over- and underestimation occurred equally in all groups.

Conclusion-DE inaccurately estimates right ventricular pressure in children with elevated right heart pressure. It should not be relied upon as the sole method of assessing right heart hemodynamics in children with RV hypertension.

(C) 2013 American Society of Echocardiography. Published by Mosby, Inc. All rights reserved. Correspondence to: Gautam K. Singh, M.D., One Children's Place, Campus Box 8116-NWT, St. Louis, MO 63132, Phone: 314-454-6095, Fax: 314-454-2561, singh_g@kids.wustl.edu.

Disclosures: None

Publisher's Disclaimer: This is a PDF file of an unedited manuscript that has been accepted for publication. As a service to our customers we are providing this early version of the manuscript. The manuscript will undergo copyediting, typesetting, and review of the resulting proof before it is published in its final citable form. Please note that during the production process errors may be discovered which could affect the content, and all legal disclaimers that apply to the journal pertain. 


\section{Keywords}

Right Ventricle; Doppler Echocardiography; Tricuspid Regurgitation; Children

\section{Introduction}

A wide variety of cardiopulmonary disease processes can lead to elevation of right heart pressure. In broad categories, elevation of right ventricular (RV) pressure can result from $\mathrm{RV}$ outflow tract obstruction, branch pulmonary artery obstruction, pulmonary vascular obstructive disease, pulmonary venous obstruction, or left ventricular inflow obstruction. ${ }^{1}$ Right heart catheterization (RHC) remains the gold standard for evaluating right heart hemodynamics in these patients. ${ }^{2,3}$ The risk of exposure to radiation and the invasive nature of RHC prompted investigators in the 1980s to introduce non-invasive methods of assessing right heart hemodynamics, specifically Doppler echocardiography (DE). These studies showed excellent correlation between DE estimated RV pressure using tricuspid valve regurgitation (TR) and direct RHC measured RV pressure. ${ }^{4-8}$ Due to its widespread availability and non-invasive nature, DE is now a widely used surrogate for RHC to determine and monitor right heart pressure in a wide variety of disease processes. ${ }^{9-16}$

Recently, the accuracy of DE in estimating right heart hemodynamics has come into question. ${ }^{17,18}$ The initial studies comparing DE and RHC to assess right heart hemodynamics utilized correlation and regression analysis. ${ }^{4-7}$ This type of statistical analysis has its shortcomings as highlighted by Bland and Altman in their seminal paper in 1986. Bland and Altman argued that a high degree of correlation between two methods of measurement should not be interpreted as indicating accuracy and interchangeability of the two methods. Instead, they advocated using bias and precision statistics to compare different techniques of measuring the same physiologic variable. ${ }^{19}$ The Bland-Altman analysis is now the accepted statistical method of comparing two methods of measuring the same physiologic variable, superseding correlation and regression. ${ }^{20}$ Recent studies in adults comparing DE and RHC using Bland-Altman analyses have suggested that DE is not as accurate as previously described for the assessment of right heart hemodynamics. ${ }^{17,} 18,21 \mathrm{~A}$ prospective, simultaneous study comparing DE and RHC using Bland-Altman analyses has not been performed in children.

In a prospective, simultaneous DE-RHC study in children, we sought to analyze the accuracy of DE in estimating right heart pressure. We further sought to determine if the degree of RV hypertension affects the accuracy of DE in assessing right heart pressure.

\section{Methods}

\section{Patient Population}

All children aged 0-17.9 years old who presented to the St. Louis Children's Hospital cardiac catheterization laboratory for a clinically indicated RHC between November 2011 and November 2012 were considered for enrollment. Inclusion criteria were two-ventricle physiology, adequate TR envelope on pre-catheterization echocardiogram, and sinus rhythm. Exclusion criteria were single ventricle physiology, presence of a subpulmonary morphologic left ventricle, and presence of a mechanical tricuspid valve. Informed, written parental consent was obtained for all subjects. Informed, written assent was obtained for all subjects $>12$ years of age. The study was approved by the Institutional Review Board for human studies at Washington University School of Medicine. 


\section{Catheterization Data}

All patients underwent simultaneous measurement of the peak RV to right atrium (RA) pressure gradient $(\triangle \mathrm{P})$ by RHC and by DE. All data collection was done with the patient in the supine position and under the usual sterile conditions of the catheterization laboratory. RV and RA pressure tracings were recorded using a standard fluid-filled, end-hole catheter in pullback fashion. $\mathrm{RV}$ and RA pressure tracings were recorded simultaneous to performance of $\mathrm{DE}$. The RHC measured $\Delta \mathrm{P}\left(\Delta \mathrm{P}_{\mathrm{C}}\right)$ was calculated by subtracting the RA Vwave pressure from the peak RV systolic pressure (RVSP) at end-expiration. Cardiac output was calculated using the Fick method 22 and indexed to body surface area. Simultaneous systemic blood pressure was obtained by arterial catheter in patients where arterial access was clinically indicated and by upper extremity non-invasive cuff measurement in those where arterial access was not clinically indicated. Interpretation of RHC data was done by a single, experienced interventional cardiologist (JJM) to exclude interobserver variability. The interventional cardiologist was blinded to the patients' diagnoses and DE data.

\section{Echocardiography Data}

Two-dimensional and color-DE was performed using commercially available ultrasound equipment (Vivid 9, GE Medical Systems, Milwaukee, Wisconsin). With the patient in the supine position, continuous-wave Doppler recordings of the maximal TR jet velocity (V) were obtained in standard apical or parasternal windows (whichever produced the highest velocity signal). The Doppler beam was kept in parallel alignment with the direction of the TR jet as visualized by color Doppler. The majority (80\%) of echocardiographic studies were performed by a single cardiac sonographer. The remainder of the studies was performed by two additional cardiac sonographers. No saline or contrast injection was used to enhance weak signals. Patients were excluded from the study if there was no measurable TR by color Doppler at the time of data acquisition. DE estimated $\Delta \mathrm{P}\left(\Delta \mathrm{P}_{\mathrm{E}}\right)$ was calculated using the modified Bernoulli equation, $\Delta \mathrm{P}_{\mathrm{E}}=4 \mathrm{~V}^{2}$. An average of three consecutive cardiac cycles was used to account for respiratory variation. Interpretation of DE data was done by a single, experienced echocardiographer (GKS) to exclude interobserver variability. The echocardiographer was blinded to the patients' diagnoses and RHC data.

\section{Assessment of TR envelope quality}

To minimize the effect of TR spectral envelope quality on the accuracy of DE, the data was analyzed using only patients with optimal TR envelopes. A TR envelope was deemed "optimal" by the interpreting cardiologist (GKS) when the spectral Doppler envelope edge was clearly identifiable in at least the first $3 / 4$ of systole. Optimal envelopes had a clear, rounded edge clearly demonstrating the peak of the TR velocity. The remainders were deemed "suboptimal" and were not included in data analysis. Examples of TR envelope quality are shown in Figure 1. An independent assessment of TR envelope quality was also done in 20 randomly selected patients by a separate, blinded observer (GKG) and interobserver reliability was assessed using Cohen's kappa method.

\section{Stratification of patients based on RV pressure}

Patients were categorized based on the ratio between RHC measured RVSP and simultaneously measured systemic systolic blood pressure (SBP). In patients with left heart obstruction, a simultaneous catheter measured left ventricular systolic pressure was used as the SBP. Two clinically relevant thresholds were used to define RV hypertension and subsequently categorize patients: (1) RVSP $\geq 1 / 2 \mathrm{SBP}$ and (2) RVSP $\geq 2 / 3 \mathrm{SBP}^{1,}{ }^{1,23}$ Group 1 included patients with RVSP $<1 / 2$ SBP and group 2 included patients with RVSP $\geq 1 / 2$ SBP. Group 3 included patients with RVSP $<2 / 3$ SBP and group 4 included patients with RVSP $\geq 2 / 3 \mathrm{SBP}$. 


\section{Statistical Analysis}

Values for descriptive statistics are reported as mean \pm SD for normally distributed data and median (interquartile range) for non-normally distributed data. Correlation coefficients between RHC and DE-derived pressures were calculated using the Pearson correlation method and the relationship of the methods was assessed using linear regression. Agreement between methods was assessed using Bland-Altman analysis. ${ }^{19}$ Accuracy of DE measurements was predefined as $95 \%$ limits of agreement within $\pm 10 \mathrm{mmHg}$. Fisher's exact test was used to compare categorical data between groups. Receiver operating characteristic (ROC) analysis was used to assess the associations between high RVSP (the reference standard) and $\Delta \mathrm{P}_{\mathrm{E}}$. The previously used clinically relevant thresholds were used to define RV hypertension: (1) RVSP $\geq 1 / 2 \mathrm{SBP}$ and (2) RVSP $\geq 2 / 3$ SBP. Binomial exact confidence intervals were calculated for the area under the curve (AUC). The Youden index was used to determine on the ROC curve the point maximizing the difference between the true positive ratio and the false positive ratio. Bootstrapped (1000 replications) confidence intervals were calculated for this point (criterion value). Based upon the criterion value, sensitivity, specificity, positive predictive value, negative predictive value, and their $95 \%$ confidence intervals were calculated. For the calculation of positive and negative predictive values, the ratio of cases in the positive and negative groups was used to represent disease prevalence. P-values $<0.05$ were considered statistically significant. Statistical analyses were performed with SPSS version 19 (SPSS Incorporated, Chicago, IL), JMP Statistical Software Release 10.0.0 (SAS Institute, Inc., Cary, NC), and MedCalc Statistics for Biomedical Research version 12.3.0.0 (MedCalc Software, Mariakerke, Belgium).

\section{Results}

\section{Patient Characteristics}

A total of 395 patients aged 0-17.9 years underwent medically indicated RHC during the study period. Forty patients were excluded due to single ventricle physiology, 2 patients were excluded with mechanical tricuspid valve and 2 patients were excluded due to subpulmonary morphologic left ventricle. Of the remaining 351 patients, $153 \mathrm{had}$ measurable TR on pre-catheterization echocardiogram and were deemed eligible for enrollment. Ten patients declined to participate and 19 patients were unable to be consented in time to participate. A total of 124 patients with a wide variety of cardiopulmonary disease processes were consented for the study. DE images were unable to be acquired in 8 patients due to hemodynamic instability at the time of RHC and in 12 patients due to logistic difficulties. A total of 104 patients underwent simultaneous RHC and DE. Four patients had no detectable TR at the time of RHC. Twenty patients were deemed to have suboptimal TR envelopes and were excluded. A total of 80 patients had simultaneous RHC and DE data obtained and analyzed. The median age was 5.5 years (1.6-10.4 years). Patient demographic data are summarized in Table 1. The RHC measured RVSP ranged from 17 to $89 \mathrm{mmHg}$ and RA V-wave pressure ranged from 4 to $20 \mathrm{mmHg}$. The DE measured maximal TR jet velocity ranged from 1.7 to $5 \mathrm{~m} / \mathrm{s}$. The $\Delta \mathrm{P}_{\mathrm{C}}$ ranged from 6 to $81 \mathrm{mmHg}$ and the $\Delta \mathrm{P}_{\mathrm{E}}$ ranged from 11 to $100 \mathrm{mmHg}$. Baseline hemodynamic data are summarized in Table 2.

\section{Assessment of TR envelope quality}

Twenty patients were deemed to have suboptimal TR envelopes (defined as less than 3/4 of systole filled) and were excluded from data analysis. Excellent interobserver reliability was seen in the determination of TR envelope quality (kappa $=0.83, \mathrm{p}<0.001$ ). Examples of TR envelope quality are shown in Figure 1. 


\section{Stratification of patients based on RV pressure}

A total of 80 patients were categorized based on the RVSP/SBP ratio. Group 1 included 43 patients with RVSP $<1 / 2 \mathrm{SBP}$, group 2 included 37 patients with RVSP $\geq 1 / 2 \mathrm{SBP}$, group 3 included 56 patients with RVSP $<2 / 3 \mathrm{SBP}$, and group 4 included 24 patients with RVSP $22 / 3$ SBP.

\section{Accuracy of DE in estimating $R V$ pressure}

Correlation between $\Delta \mathrm{P}_{\mathrm{C}}$ and $\Delta \mathrm{P}_{\mathrm{E}}$ was reasonable in all groups (group 1: $\mathrm{R}=0.8$; group 2: $\mathrm{R}=0.81$, group 3: $\mathrm{R}=0.95$; group $4: \mathrm{R}=0.75, \mathrm{p}<0.001$ ) (Figure 2). Agreement between the two methods was good in group 1 (bias $3.07 \mathrm{mmHg}, 95 \%$ limits of agreement +9.66 to $-3.52 \mathrm{mmHg}$ ), but poor in group 2 (bias $1.49 \mathrm{mmHg}, 95 \%$ limits of agreement +22.43 to $-19.45 \mathrm{mmHg}$ ), and good in group 3 (bias $2.5 \mathrm{mmHg}$, $95 \%$ limits of agreement +10.18 to $-4.5 \mathrm{mmHg}$ ) but poor in group 4 (bias $1.16 \mathrm{mmHg}, 95 \%$ limits of agreement +26.41 to $-24.09 \mathrm{mmHg}$ ) (Figure 3).

With accuracy predefined as $95 \%$ limits of agreement $\pm 10 \mathrm{mmHg}$, DE was inaccurate in $1 / 43(2 \%)$ patients in group 1 versus $9 / 37(24 \%)$ patients in group 2 and inaccurate in 1/56 (2\%) patients in group 3 versus 8/24 (33\%) patients in group 4. An example of the inaccuracy of DE is shown in Figure 4. Over and under-estimation occurred equally in all groups.

\section{Clinical implications}

To determine the clinical relevance of our findings, we calculated a DE derived RVSP/SBP ratio and compared our findings with the true RHC determined RVSP/SBP ratio using the same two clinical relevant cutoffs used previously. The DE derived RVSP (RVSP ${ }_{E}$ ) was calculated using $\Delta \mathrm{P}_{\mathrm{E}}+\mathrm{RHC}$ measured RA V-wave pressure. In the $\mathrm{RVSP}_{\mathrm{E}} \geq 1 / 2 \mathrm{SBP}$ group, $\mathrm{RVSP}_{\mathrm{E}} / \mathrm{SBP}$ ratio misclassified $14 / 80$ patients (18\%). Eight were underclassified and 6 were overclassified. In the $\mathrm{RVSP}_{\mathrm{E}} \geq 2 / 3 \mathrm{SBP}$ group, the $\mathrm{RVSP}_{\mathrm{E}} / \mathrm{SBP}$ ratio misclassified 16/80 patients (20\%). Eight were underclassified and 8 were overclassified.

\section{Sensitivity, specificity, and predictive value of DE}

ROC analysis was used to assess the associations between high RVSP (the reference standard) and $\triangle \mathrm{P}_{\mathrm{E}}$. Two clinically relevant thresholds were used to define $\mathrm{RV}$ hypertension: (1) RVSP $\geq 1 / 2 \mathrm{SBP}$ and (2) RVSP $\geq 2 / 3 \mathrm{SBP}$. Using RVSP $\geq 1 / 2 \mathrm{SBP}$ as the threshold, there was an AUC of 0.93 (95\% CI 0.85-0.97, $\mathrm{p}<0.001$ ). A $\Delta \mathrm{P}_{\mathrm{E}}$ of $>28 \mathrm{mmHg}$ was $82 \%$ sensitive and $89 \%$ specific in detecting an RVSP $\geq 1 / 2 \mathrm{SBP}$, with an $86 \%$ positive predictive value and $86 \%$ negative predictive value. Using RVSP $\geq 2 / 3 \mathrm{SBP}$ as the threshold, there was an AUC of 0.91 (95\% CI $0.84-0.96, \mathrm{p}<0.001)$. A $\Delta \mathrm{P}_{\mathrm{E}}$ of $>35.5 \mathrm{mmHg}$ was $81 \%$ sensitive and $88 \%$ specific in detecting an RVSP $\geq 2 / 3 \mathrm{SBP}$, with an $81 \%$ positive predictive value and $88 \%$ negative predictive value (Figure 5).

\section{Discussion}

In this study we evaluated the accuracy of DE in predicting simultaneously measured RV pressure by RHC in pediatric patients. Our study population had a wide variety of cardiopulmonary disease processes leading to a wide range of RV pressures. We have shown for the first time that the degree of RV hypertension affects the accuracy of DE in estimating right heart pressure.

Using predefined accuracy limits of $95 \%$ limits of agreement within bias $\pm 10 \mathrm{mmHg}, \mathrm{DE}$ was inaccurate in estimating RHC measured RVSP in $24 \%$ of children with RVSP $\geq 1 / 2$ systemic. DE became more frequently inaccurate with increasing RV pressure and was 
inaccurate in $33 \%$ of children with RVSP $\geq 2 / 3$ systemic. Furthermore, the direction of inaccuracy was unpredictable, with both over- and underestimation occurring equally. From a clinical and diagnostic perspective, our DE derived RVSP misclassified patients approximately $20 \%$ of the time, depending on which RVSP/SBP ratio cutoff was used. Since our method of generating a DE derived RVSP was done by adding an actual RHC measured RA pressure to the DE measured RV-RA pressure gradient, our incidence of misclassification is likely underestimated. In actual clinical practice, a true RA pressure is unavailable and an estimated RA pressure is typically used. This would more than likely lead to increased error in the DE estimated RVSP and a higher incidence of misclassification. This was seen in the study by Fisher et al, where pulmonary hypertension severity was misclassified in almost $50 \%$ of patients when using an estimated RA pressure to generate a DE estimated pulmonary artery systolic pressure (PASP). ${ }^{18}$

The clinical implications of these findings are significant, as DE is widely used to screen for and manage many different causes of RV hypertension. RV hypertension has previously been defined as RVSP $>1 / 2$ systemic levels and it is a common clinical practice to refer children for invasive cardiac catheterization once DE shows an estimated RV pressure of > 1/2 to 2/3 systemic levels. ${ }^{1}$ An American Heart Association class II indication exists for RHC with balloon angioplasty in children with pulmonary artery obstruction and RVSP $>2 / 3$ systemic pressure. ${ }^{23}$ Both American College of Cardiology/American Heart Association and American Society of Echocardiography guidelines for clinical use of echocardiography 24,25 recommend the use of serial echocardiography in pediatric patients to monitor hemodynamic adaptation to surgical or transcatheter repair or palliation in order to identify recurrence of abnormalities. Such longitudinal monitoring allows for proactive surgical, transcatheter, or medical intervention. Our study questions the accuracy and predictive value of $\mathrm{DE}$ as a solo monitoring tool in these situations.

Our results extend the findings of recent studies demonstrating the overall inaccuracy of DE in estimating right heart pressures in adult patients with pulmonary hypertension ${ }^{17,18,21}$ to a strictly pediatric population with a broad range of diagnoses. Importantly, the aforementioned studies did not stratify patients based on RV pressure, which we have done. Skinner et al simultaneously compared DE and RHC for assessment of right heart hemodynamics in 26 infants and found that in patients with RV systolic pressure $>60 \mathrm{mmHg}$, the accuracy of DE diminished. ${ }^{26}$ Our study confirms this finding in a larger, more robust pediatric patient population. Furthermore, previous studies highlighting the inaccuracy of DE have measured PASP. ${ }^{17,}{ }^{18}$ In order to report an estimated PASP by DE, RA pressure was estimated non-invasively in these studies. While echocardiographic methods of estimating RA pressure have previously been validated in adults ${ }^{27}$, these methods have not been well studied in children and have more recently come into question. ${ }^{28}$ Rich et al found that the estimation of RA pressure was an important source of error in the DE estimated PASP and may have contributed to the inaccuracy of DE that they reported. ${ }^{17} \mathrm{We}$ avoided this important source of error by comparing the RV to RA pressure drop by both DE and RHC.

Several explanations exist as to why a discrepancy between DE and RHC measurements may be seen. First, using the Bernoulli equation to estimate pressure gradient between two chambers assumes a perfect, parallel alignment between the ultrasound beam and the maximal regurgitant jet. ${ }^{29}$ Small deviations in angle $\left(<20^{\circ}\right)$ may produce mild errors $(<10 \%)$ in velocity measurements, which may be acceptable in low velocity flows. However, when the Doppler is used to obtain pressure gradients, even a small error in velocity can produce significant discrepancy in the gradient due to the quadratic relationship between velocity and pressure gradient. ${ }^{30}$ In our study, the TR jet was visualized by colorflow mapping in order to ensure that the angle between the Doppler beam and TR jet was $<20^{\circ}$ for all patients. The 
TR jet is a three dimensional phenomenon and using two dimensional echocardiography to visualize the TR jet assumes that the maximal TR jet is able to be captured in the two dimensional imaging plane..$^{30}$ It is possible that the actual maximal TR jet is outside the imaging plane when imaging in only two dimensions. This limitation could account for the inaccuracy of using 2D echocardiography to estimate right heart pressures. Furthermore, the modified Bernoulli equation assumes negligible viscous and inertial forces, as well as complete transformation of potential energy (pressure in the RV) to kinetic energy (the TR jet). Giardini et al demonstrated in an in vitro flow model of atrioventricular valve regurgitation that conditions commonly seen in clinical practice can lead to incomplete transformation of potential energy to kinetic energy. They showed that an eccentric TR jet may interact with the RA wall (Coanda effect) causing viscous losses. As well, increasing hematocrit, frequently encountered in patients with cyanotic congenital heart disease, can lead to viscous losses. Such viscous losses are unaccounted for in the modified Bernoulli equation, and can lead to underestimation of RV pressure. Finally, they suggested that in children, the absolute RA size is small and inertial forces unaccounted for by the modified Bernoulli equation can cause pressure recovery in the receiving chamber, leading to overestimation of RV pressure by DE. ${ }^{31}$ All of these factors can lead to both over and underestimation, as was seen in our study population.

The most plausible explanation for diminished accuracy of DE with increasing RV pressures is inherent to the Bernoulli formula itself. While performing DE, each increment of error in velocity measurement is squared and multiplied by four when applying the Bernoulli equation. Thus, it stands to reason that as the TR jet velocity increases, the calculated $\Delta \mathrm{P}_{\mathrm{E}}$ can differ from the $\Delta \mathrm{P}_{\mathrm{C}}$ by a larger increment. Skinner et al suggested it might be better to express TR in terms of velocity $(\mathrm{m} / \mathrm{s})$ rather than pressure drop $(\mathrm{mmHg})$ to minimize this source of error ${ }^{26}$ However, pressure drop expressed in $\mathrm{mmHg}$ remains the accepted parameter in clinical practice today. The lack of agreement between DE and RHC seen in our study is likely a result of a combination of the inherent limitations of the simplified Bernoulli equation as well as limitations in study design, which are addressed below. Despite its limitations, echocardiography remains the most widely available and least invasive method for assessing right heart hemodynamics and function in children with RV hypertension. Our study highlights the importance of combining TR estimated RV pressure with other echocardiographic parameters of RV pressure evaluation when making clinical decisions regarding diagnosis and management of patients with RV hypertension. Such parameters may include RA enlargement, RV dilation/hypertrophy, interventricular septal configuration/motion, main pulmonary artery dilation, and pulmonary artery acceleration time. ${ }^{1,32,33}$ In the absence of other supporting evidence of RV pressure overload, the TR jet alone should be used with caution.

\section{Limitations}

Our study population was heterogeneous and subgroups of patients based on diagnoses and level of right heart obstruction were small. This precluded adequate subgroup analyses to test whether the level of right heart obstruction affects accuracy of DE. Jarcut et al recently showed that at similar levels RV pressure overload, patients with pulmonary valve stenosis had less RV dilation and better RV performance compared to those with pulmonary arterial hypertension. ${ }^{34}$ This suggests that perhaps the nature of right heart obstruction affects right heart hemodynamics and function, which could affect the accuracy of DE in predicting RV pressures. Our study was also performed at one point in time, and therefore does not address accuracy of serial DE measures over time or test-retest variability. 


\section{Limitations of Study Design}

Acquisition of DE data was limited by technical difficulties encountered during simultaneous DE and RHC. All DE data was obtained with patients in the supine position. Inability to change patient position may have affected acoustic window quality, particularly in apical views in older patients. Additionally, parasternal windows were difficult to obtain in some patients due to the position of sterile drapes, which impeded optimal probe position. These limitations may have led to inability to capture the highest velocity TR jet, contributing to out of plane error. DE was performed by three different cardiac sonographers, which raises the possibility of inter-observer variability in image acquisition. However, $80 \%$ of the studies were done by a single cardiac sonographer, minimizing the impact of this source of error. Finally, for the calculation of positive and negative predictive values, the ratio of cases in the positive and negative groups of our study population was used to represent disease prevalence. This does not represent true RV hypertension disease prevalence, as our study population consists of only children referred for RHC. Therefore, the prevalence of $\mathrm{RV}$ hypertension in our study population is almost certainly higher than in the general population. The true PPV of DE for detecting RV hypertension in the general population is likely lower and the true NPV likely higher than what we report.

\section{Conclusions}

This study is the first to show that DE estimates of RV pressure in children are not completely accurate, specifically in children with elevated right heart pressure. Significantly, both over and underestimation occur. DE may not be interchangeable with RHC in determining right heart pressure in children with RV hypertension. DE should not be relied upon as the sole method of diagnosing and managing children with elevated right heart pressure. Continued development of reliable, non-invasive techniques to assess right heart hemodynamics in children is warranted.

\section{Acknowledgments}

This study was supported by the following grants: Pediatric Physician Scientist Training Grant (NIH 5 T32 HD043010-09) and Postdoctoral Mentored Training Program in Clinical Investigation (NIH UL1 TR000448).

\section{Abbreviations}

$\begin{array}{ll}\text { DE } & \text { Doppler echocardiography } \\ \text { RHC } & \text { right heart catheterization } \\ \text { RVSP } & \text { catheterization measured right ventricular systolic pressure } \\ \text { SBP } & \text { systemic systolic blood pressure } \\ \text { RA } & \text { right atrium } \\ \text { RV } & \text { right ventricle } \\ \Delta \mathbf{P c} & \text { catheterization measured right ventricle to right atrium pressure gradient } \\ \Delta \mathbf{P}_{\mathbf{E}} & \text { echocardiography estimated right ventricle to right atrium pressure gradient } \\ \text { TR } & \text { tricuspid regurgitation } \\ \text { ROC } & \text { receiver operating curve } \\ \text { PASP } & \text { pulmonary artery systolic pressure }\end{array}$




\section{References}

1. King ME, Braun H, Goldblatt A, Liberthson R, Weyman AE. Interventricular septal configuration as a predictor of right ventricular systolic hypertension in children: A cross-sectional echocardiographic study. Circulation. 1983; 68:68-75. [PubMed: 6851056]

2. Galie N, Hoeper MM, Humbert M, Torbicki A, Vachiery JL, Barbera JA, et al. Guidelines for the diagnosis and treatment of pulmonary hypertension: The task force for the diagnosis and treatment of pulmonary hypertension of the european society of cardiology (esc) and the european respiratory society (ers), endorsed by the international society of heart and lung transplantation (ishlt). European heart journal. 2009; 30:2493-2537. [PubMed: 19713419]

3. McLaughlin VV, Archer SL, Badesch DB, Barst RJ, Farber HW, Lindner JR, et al. Accf/aha 2009 expert consensus document on pulmonary hypertension: A report of the american college of cardiology foundation task force on expert consensus documents and the american heart association: Developed in collaboration with the american college of chest physicians, american thoracic society, inc., and the pulmonary hypertension association. Circulation. 2009; 119:2250-2294. [PubMed: 19332472]

4. Yock PG, Popp RL. Noninvasive estimation of right ventricular systolic pressure by doppler ultrasound in patients with tricuspid regurgitation. Circulation. 1984; 70:657-662. [PubMed: 6478568]

5. Currie PJ, Seward JB, Chan KL, Fyfe DA, Hagler DJ, Mair DD, et al. Continuous wave doppler determination of right ventricular pressure: A simultaneous doppler-catheterization study in 127 patients. Journal of the American College of Cardiology. 1985; 6:750-756. [PubMed: 4031289]

6. Berger M, Haimowitz A, Van Tosh A, Berdoff RL, Goldberg E. Quantitative assessment of pulmonary hypertension in patients with tricuspid regurgitation using continuous wave doppler ultrasound. Journal of the American College of Cardiology. 1985; 6:359-365. [PubMed: 4019921]

7. Hecht SR, Berger M, Berdoff RL, Van Tosh A, Stimola JM. Use of continuous-wave doppler ultrasound to evaluate and manage primary pulmonary hypertension. Chest. 1986; 90:781-783. [PubMed: 3769591]

8. Hatle L, Angelsen BA, Tromsdal A. Non-invasive estimation of pulmonary artery systolic pressure with doppler ultrasound. British heart journal. 1981; 45:157-165. [PubMed: 7459175]

9. Bossone E, Bodini BD, Mazza A, Allegra L. Pulmonary arterial hypertension: The key role of echocardiography. Chest. 2005; 127:1836-1843. [PubMed: 15888866]

10. Lanzarini L, Fontana A, Lucca E, Campana C, Klersy C. Noninvasive estimation of both systolic and diastolic pulmonary artery pressure from doppler analysis of tricuspid regurgitant velocity spectrum in patients with chronic heart failure. American heart journal. 2002; 144:1087-1094. [PubMed: 12486435]

11. Hachulla E, Gressin V, Guillevin L, Carpentier P, Diot E, Sibilia J, et al. Early detection of pulmonary arterial hypertension in systemic sclerosis: A french nationwide prospective multicenter study. Arthritis and rheumatism. 2005; 52:3792-3800. [PubMed: 16320330]

12. Casaclang-Verzosa G, McCully RB, Oh JK, Miller FA Jr, McGregor CG. Effects of pulmonary thromboendarterectomy on right-sided echocardiographic parameters in patients with chronic thromboembolic pulmonary hypertension. Mayo Clinic proceedings. Mayo Clinic. 2006; 81:777782 .

13. Slaughter JL, Pakrashi T, Jones DE, South AP, Shah TA. Echocardiographic detection of pulmonary hypertension in extremely low birth weight infants with bronchopulmonary dysplasia requiring prolonged positive pressure ventilation. Journal of perinatology: official journal of the California Perinatal Association. 2011; 31:635-640. [PubMed: 21311503]

14. Mourani PM, Sontag MK, Younoszai A, Ivy DD, Abman SH. Clinical utility of echocardiography for the diagnosis and management of pulmonary vascular disease in young children with chronic lung disease. Pediatrics. 2008; 121:317-325. [PubMed: 18245423]

15. Rosenzweig EB, Feinstein JA, Humpl T, Ivy DD. Pulmonary arterial hypertension in children: Diagnostic work-up and challenges. Progress in pediatric cardiology. 2009; 27:4-11. [PubMed: 21691442] 
16. Parent F, Bachir D, Inamo J, Lionnet F, Driss F, Loko G, et al. A hemodynamic study of pulmonary hypertension in sickle cell disease. The New England journal of medicine. 2011; 365:44-53. [PubMed: 21732836]

17. Rich JD, Shah SJ, Swamy RS, Kamp A, Rich S. Inaccuracy of doppler echocardiographic estimates of pulmonary artery pressures in patients with pulmonary hypertension: Implications for clinical practice. Chest. 2011; 139:988-993. [PubMed: 20864617]

18. Fisher MR, Forfia PR, Chamera E, Housten-Harris T, Champion HC, Girgis RE, et al. Accuracy of doppler echocardiography in the hemodynamic assessment of pulmonary hypertension. American journal of respiratory and critical care medicine. 2009; 179:615-621. [PubMed: 19164700]

19. Bland JM, Altman DG. Statistical methods for assessing agreement between two methods of clinical measurement. Lancet. 1986; 1:307-310. [PubMed: 2868172]

20. Critchley LA, Critchley JA. A meta-analysis of studies using bias and precision statistics to compare cardiac output measurement techniques. Journal of clinical monitoring and computing. 1999; 15:85-91. [PubMed: 12578081]

21. Arcasoy SM, Christie JD, Ferrari VA, Sutton MS, Zisman DA, Blumenthal NP, et al. Echocardiographic assessment of pulmonary hypertension in patients with advanced lung disease. American journal of respiratory and critical care medicine. 2003; 167:735-740. [PubMed: 12480614]

22. McMichael J, Sharpey-Schafer EP. Cardiac output in man by a direct fick method: Effects of posture, venous pressure change, atropine, and adrenaline. British heart journal. 1944; 6:33-40. [PubMed: 18609953]

23. Feltes TF, Bacha E, Beekman RH 3rd, Cheatham JP, Feinstein JA, Gomes AS, et al. Indications for cardiac catheterization and intervention in pediatric cardiac disease: A scientific statement from the american heart association. Circulation. 2011; 123:2607-2652. [PubMed: 21536996]

24. Cheitlin MD, Alpert JS, Armstrong WF, Aurigemma GP, Beller GA, Bierman FZ, et al. Acc/aha guidelines for the clinical application of echocardiography. A report of the american college of cardiology/american heart association task force on practice guidelines (committee on clinical application of echocardiography). Developed in collaboration with the american society of echocardiography. Circulation. 1997; 95:1686-1744. [PubMed: 9118558]

25. Lai WW, Geva T, Shirali GS, Frommelt PC, Humes RA, Brook MM, et al. Guidelines and standards for performance of a pediatric echocardiogram: A report from the task force of the pediatric council of the american society of echocardiography. Journal of the American Society of Echocardiography: official publication of the American Society of Echocardiography. 2006; 19:1413-1430. [PubMed: 17138024]

26. Skinner JR, Stuart AG, O’Sullivan J, Heads A, Boys RJ, Hunter S. Right heart pressure determination by doppler in infants with tricuspid regurgitation. Archives of disease in childhood. 1993; 69:216-220. [PubMed: 8215524]

27. Lang RM, Bierig M, Devereux RB, Flachskampf FA, Foster E, Pellikka PA, et al. Recommendations for chamber quantification: A report from the american society of echocardiography's guidelines and standards committee and the chamber quantification writing group, developed in conjunction with the european association of echocardiography, a branch of the european society of cardiology. Journal of the American Society of Echocardiography: official publication of the American Society of Echocardiography. 2005; 18:1440-1463. [PubMed: 16376782]

28. Brennan JM, Blair JE, Goonewardena S, Ronan A, Shah D, Vasaiwala S, et al. Reappraisal of the use of inferior vena cava for estimating right atrial pressure. Journal of the American Society of Echocardiography: official publication of the American Society of Echocardiography. 2007; 20:857-861. [PubMed: 17617312]

29. Snider, AR.; Serwer, GA.; Ritter, SB. Echocardiography in pediatric heart disease. 2. St. Louis: Mosby-Year Book, Inc; 1997.

30. Quinones MA, Otto CM, Stoddard M, Waggoner A, Zoghbi WA. Recommendations for quantification of doppler echocardiography: A report from the doppler quantification task force of the nomenclature and standards committee of the american society of echocardiography. Journal of the American Society of Echocardiography: official publication of the American Society of Echocardiography. 2002; 15:167-184. [PubMed: 11836492] 
31. Giardini A, Tacy TA. Non-invasive estimation of pressure gradients in regurgitant jets: An overdue consideration. European journal of echocardiography: the journal of the Working Group on Echocardiography of the European Society of Cardiology. 2008; 9:578-584. [PubMed: 18490278]

32. Feneley M, Gavaghan T. Paradoxical and pseudoparadoxical interventricular septal motion in patients with right ventricular volume overload. Circulation. 1986; 74:230-238. [PubMed: 3731414]

33. Yared K, Noseworthy P, Weyman AE, McCabe E, Picard MH, Baggish AL. Pulmonary artery acceleration time provides an accurate estimate of systolic pulmonary arterial pressure during transthoracic echocardiography. Journal of the American Society of Echocardiography: official publication of the American Society of Echocardiography. 2011; 24:687-692. [PubMed: 21511434]

34. Jurcut R, Giusca S, Ticulescu R, Popa E, Amzulescu MS, Ghiorghiu I, et al. Different patterns of adaptation of the right ventricle to pressure overload: A comparison between pulmonary hypertension and pulmonary stenosis. Journal of the American Society of Echocardiography: official publication of the American Society of Echocardiography. 2011; 24:1109-1117. [PubMed: 21873027] 

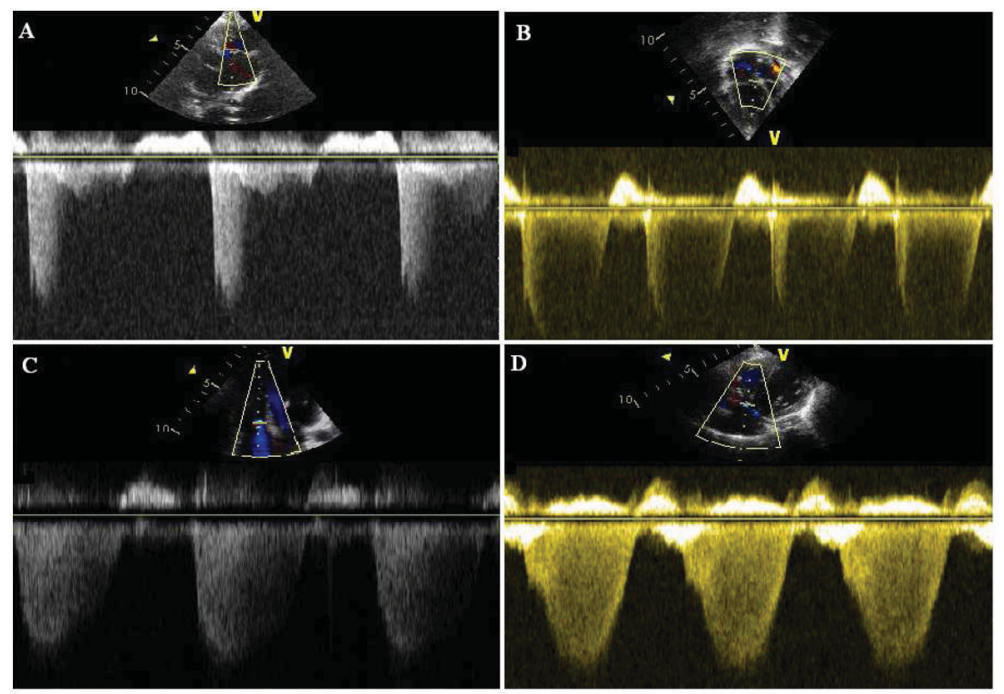

Figure 1. Tricuspid valve regurgitation envelope quality

TR envelope was deemed "optimal" if the spectral envelope was filled in at least the initial $3 / 4$ of systole with a sharp, rounded edge clearly demonstrating the peak of the TR velocity. (A) 1/2 filled (patient excluded). (B) 2/3 filled (patient excluded). (C) 3/4 filled (patient included). (D) Full (patient included). 

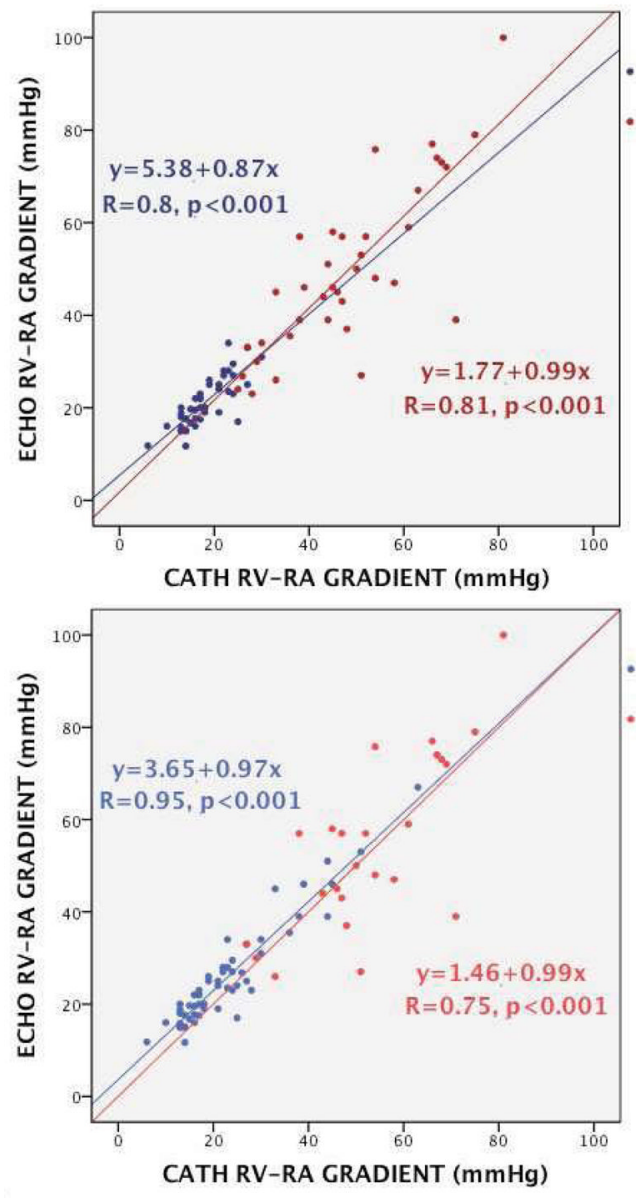

GROUP 1:

RVSP $<1 / 2 S B P$

GROUP 2:

RVSP $\geq 1 / 2 S B P$

Figure 2. Correlation between DE and RHC derived RV-RA gradient

Regression plots showing similar correlation between $\Delta \mathrm{P}_{\mathrm{E}}$ and $\Delta \mathrm{P}_{\mathrm{C}}$ in all groups. The upper plot shows correlation between group 1 and 2 and the bottom plot shows correlation between group 3 and 4 . 

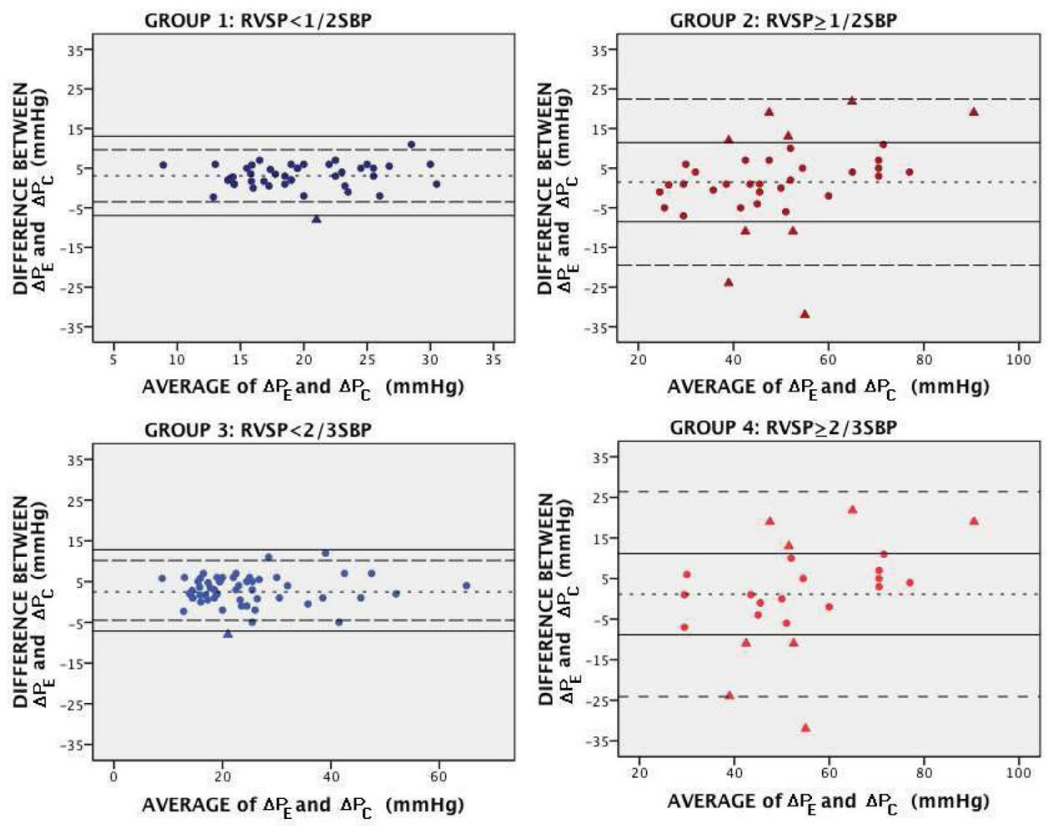

Figure 3. Bland-Altman analysis showing lack of agreement in groups with high RV pressure Bland-Altman plots showing mean bias (dotted line), 95\% limits of agreement (dashed lines), and predefined accuracy limits of bias $\pm 10 \mathrm{mmHg}$ (solid lines). In groups with elevated RV pressure ( 2 and 4 ), the $95 \%$ limits of agreement are wide and many patients fall outside the predefined accuracy limits (triangles represent inaccurate measurements). Both over- and underestimation are seen equally. 

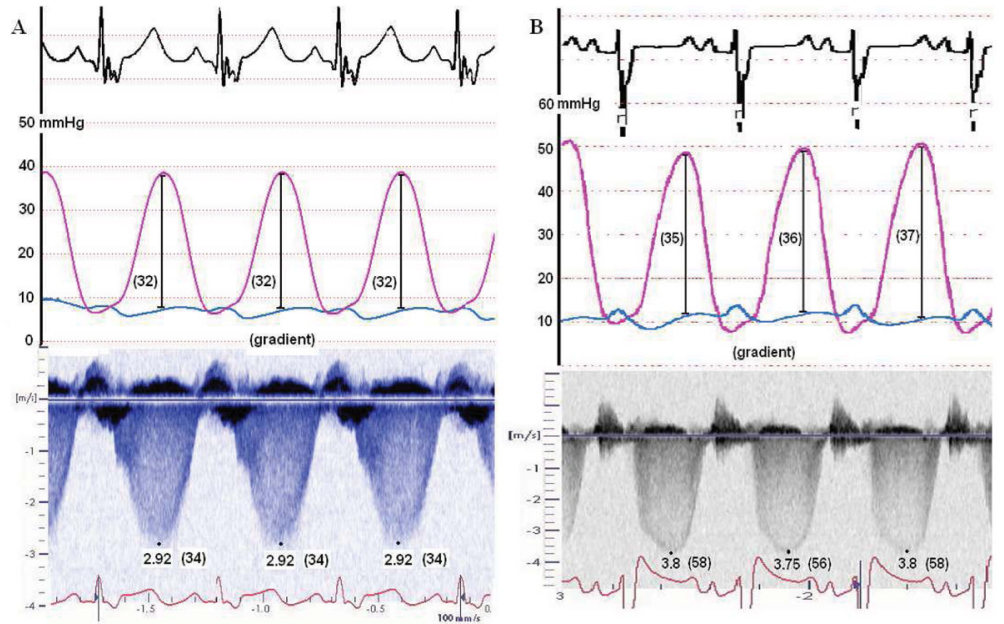

Figure 4. Inaccuracy occurs despite optimal TR envelope quality $\mathrm{DE}$ can be both accurate and inaccurate in patients with full TR envelopes. This is shown in these simultaneous DE and RHC pressure recordings. (A) Patient with accurate DE predicted RV-RA gradient and full TR envelope and (B) patient with inaccurate DE predicted RV-RA gradient and full TR envelope. 

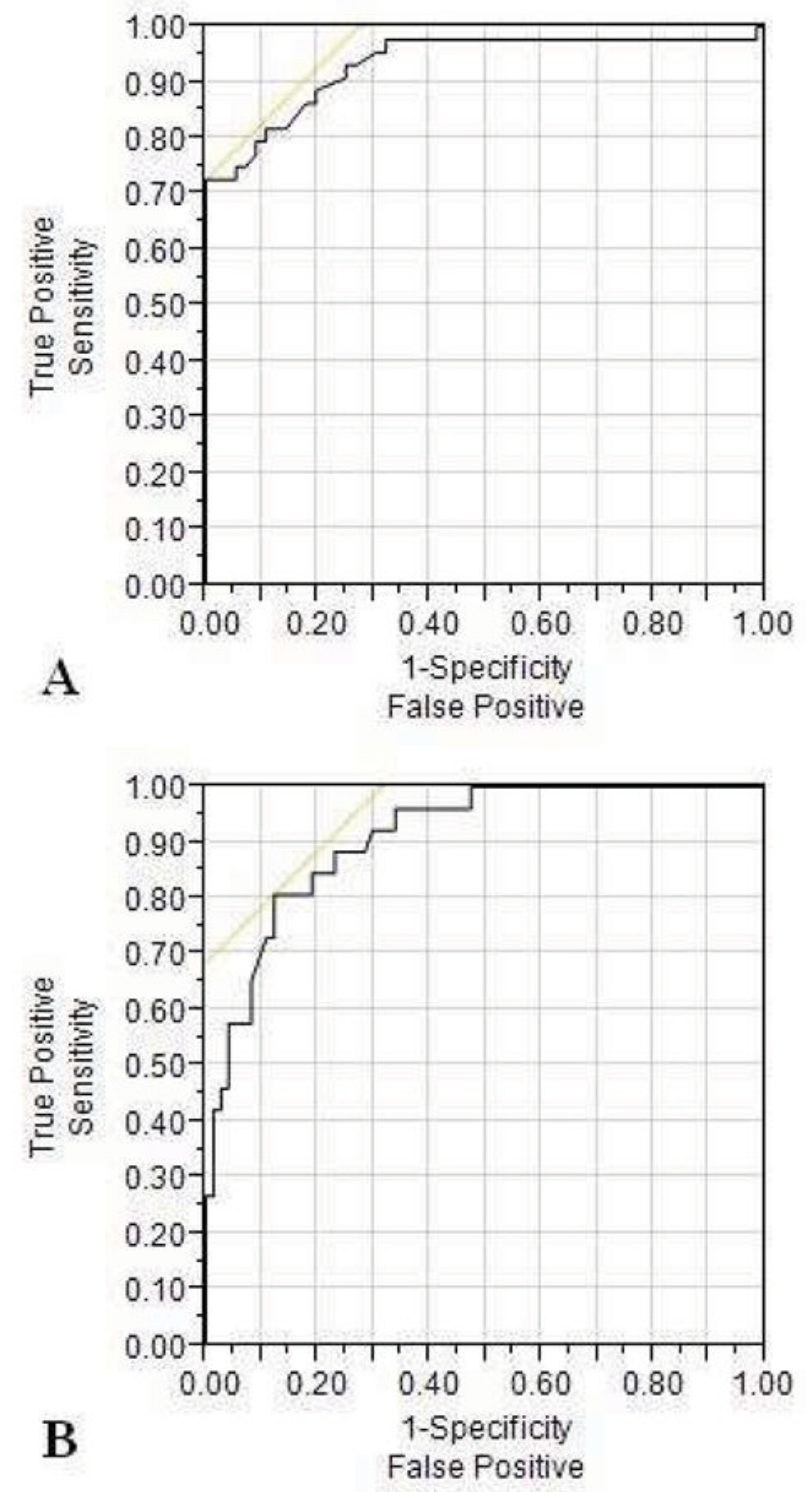

Figure 5. Receiver Operating Characteristic (ROC) curves

Receiver operative curves (ROC) showing the ability of $\Delta \mathrm{P}_{\mathrm{E}}$ to predict RV hypertension using two thresholds: (A) RVSP $\geq 1 / 2$ SBP, AUC 0.93 (95\% CI 0.85-0.97, p<0.001) and (B) RVSP $22 / 3 S B P$, AUC 0.91 (95\% CI 0.84-0.96, p<0.001). The yellow line is drawn at a 45 degree angle tangent to the ROC curve. This marks the point (criterion value) at which false negatives and false positives have similar costs. 
